This item was submitted to Loughborough's Research Repository by the author.

Items in Figshare are protected by copyright, with all rights reserved, unless otherwise indicated.

\title{
Buckling load reduction for stiffened panels due to cutouts in ribs
}

PLEASE CITE THE PUBLISHED VERSION

http://www.aiaa.org/content.cfm?pageid=318\&volume=36\&issue=2\&pubid=2\&paperid=7510

\section{PUBLISHER}

(C) American Institute of Aeronautics and Astronautics (AIAA)

\section{VERSION}

AM (Accepted Manuscript)

\section{LICENCE}

CC BY-NC-ND 4.0

\section{REPOSITORY RECORD}

Watson, Andrew, F.W. Williams, and David Kennedy. 2011. "Buckling Load Reduction for Stiffened Panels Due to Cutouts in Ribs". figshare. https://hdl.handle.net/2134/8694. 
This item was submitted to Loughborough's Institutional Repository (https://dspace.lboro.ac.uk/) by the author and is made available under the following Creative Commons Licence conditions.

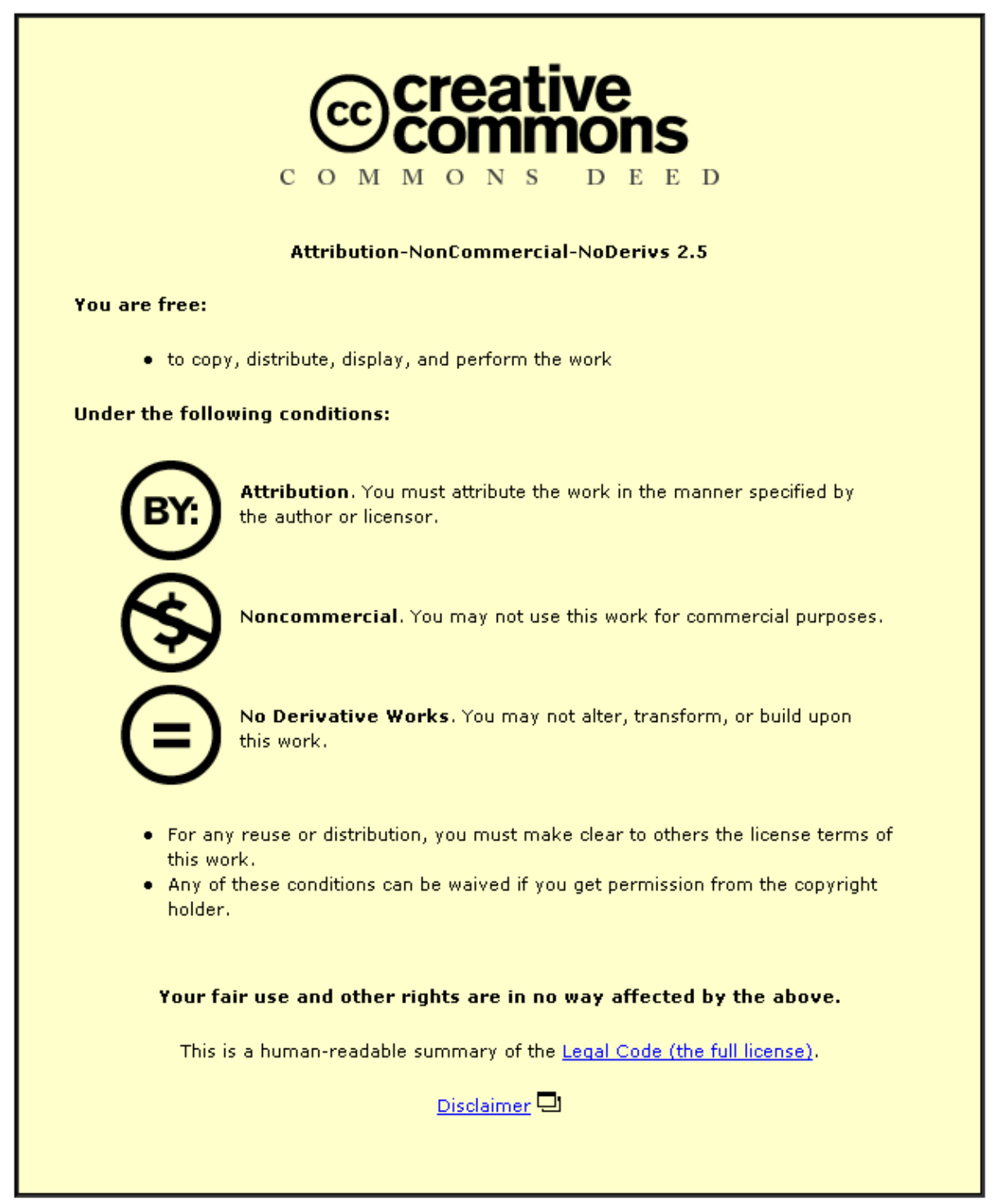

For the full text of this licence, please go to: http://creativecommons.org/licenses/by-nc-nd/2.5/ 


\title{
Buckling Load Reduction for Stiffened Panels due to Cutouts in Ribs
}

\author{
Andrew Watson*, Fred W.Williams $†$ and David Kennedy $\ddagger$ \\ Division of Structural Engineering, Cardiff School of Engineering, University of \\ Wales Cardiff, Queen’s Buildings, The Parade, P.O. Box 686, Cardiff CF2 3TB, U.K.
}

\begin{abstract}
In aerospace structures it is common to find stiffened panels with transverse supporting structures, e.g. wing ribs or fuselage frames. Incorporating cutouts into these supporting structures to allow the stringers to pass through freely considerably reduces the buckling load of the panels. It is shown that a minor modification in the fabrication of the stiffened panel gives most of the advantages of cutouts while still giving a buckling load close to that of a panel with no cutouts.
\end{abstract}

\section{Introduction}

In aerospace structures it is common to find stiffened panels with transverse supporting structures, e.g. wing ribs or fuselage frames. The aim of this parametric study is to see if cutouts in these transverse supporting structures, through which stringers pass, reduce the buckling loads or, alternatively, increase the mass when

\footnotetext{
*Research Student.

$\dagger$ Professor, Head of Structures, Member, AIAA.

$\ddagger$ Lecturer, Member, AIAA.
} 
designing. It is shown that incorporating such cutouts into the transverse supporting structure can considerably reduce the buckling load of the panel. This is done by considering the limiting case of supporting structures which completely clamp the ends of the panel except where the cutouts are.

The results are compared to those for a panel with completely clamped ends, i.e. without cutouts. The reduction in the buckling capacity is due to the lack of constraints within each cutout allowing the buckling mode to pass through it to the next bay. The presence of the cutouts means that the buckling mode does not have to repeat in every bay, unlike the case without cutouts.

\section{Modelling of Clamped Ends}

All computing is performed using VICONOPT ${ }^{1}$ (VIPASA with CONstraints and OPTimisation), which is a FORTRAN 77 computer program that incorporates the earlier programs VIPASA ${ }^{2}$ (Vibration and Instability of Plate Assemblies including Shear and Anisotropy) and VICON ${ }^{3}$ (VIPASA with CONstraints). It covers any prismatic plate assembly, i.e. panels of constant cross section, composed of anisotropic plates each of which can carry any combination of longitudinally invariant in-plane stresses. It can be used as either an analysis or an optimum design program. The VIPASA and VICON analysis features cover the calculation of eigenvalues, i.e. the critical load factors in elastic buckling problems or the natural frequencies in undamped vibration problems. The analysis is based upon the exact solution of the governing differential equations of the constituent members, yielding exact stiffness matrices of which the elements are transcendental functions of the load factor or 
frequency. The resulting transcendental eigenproblem requires an iterative solution which is performed using the Wittrick - Williams algorithm ${ }^{4}$.

VICON can be used to solve any analysis problem which could otherwise be solved by VIPASA but includes substantial additional capability as well, because VIPASA assumes modes with sinusoidal longitudinal variation of half wavelength, $\lambda$, whereas VICON modes are weighted sums of such modes for a series of values of $\lambda$.

Displacements along the infinite length of the plate assembly, of which a typical plate is shown in Fig. 1, are assumed to be the real vector $\mathrm{D}_{\mathrm{A}}$ given by

$$
\mathbf{D}_{\mathrm{A}}=\sum_{\mathrm{m}=-\infty}^{\infty} \mathbf{D}_{\mathrm{m}} \exp (2 \mathrm{i} \pi \mathrm{mx} / \mathrm{L})
$$

where $L$ is the length over which the mode repeats, $D_{m}$ is a complex displacement amplitude vector for the assumed sinusoidal longitudinal half wavelength $\lambda$, and $m$ is the number of sinusoidal waves in length $\mathrm{L}$, so that $\lambda=\mathrm{L} / 2 \mathrm{~m}$.

VICONOPT uses Lagrangian multipliers to couple the responses for an appropriate $^{3}$ set of the half wavelengths $\lambda$ of the infinitely long plate assembly so as to satisfy support conditions repeating at longitudinal intervals $l$. Thus a plate assembly of finite length $l$ with simply supported ends may be modelled reasonably accurately by representing the (continuous) transverse simple supports by a line of rigid point supports at $x=0$. The results assume that the mode repeats over a length $L=2 l / \xi$ for some value $0 \leq \xi \leq 1$. Each value of $\xi$ generates the truncated infinite series

$$
\lambda=l /(\xi+2 m) \quad(m=0, \pm 1, \pm 2, \pm 3, \pm 4 \ldots . \pm q)
$$

Here $\mathrm{q}$ is chosen to be high enough to give acceptable results. Table 1 lists the values of $\lambda$ derived from Eq. (2) for typical values of $\xi$, with negative values indicating the use of complex conjugate matrices and $\infty$ indicating a rigid body mode ${ }^{3}$. 
An appropriate model of a clamped end point support at $x=0$ for the $j$-th element $D_{A j}$ of $D_{A}$ requires, if the $j$-th element is a w displacement (see Fig. 1), that $\mathrm{w}=0$ and $\mathrm{dw} / \mathrm{dx}=0$. After differentiating Eq. (1), this gives the constraints as

$$
\begin{aligned}
& \sum \mathrm{D}_{\mathrm{mj}}=0 \\
& \sum \mathrm{mD}_{\mathrm{mj}}=0
\end{aligned}
$$

\section{Modelling of the Cutouts}

To model the cutouts in the transverse supporting structure shown in Fig. 2, which repeats at longitudinal intervals of $l$, it is assumed that the supporting structure is very stiff and behaves as a clamped support in the limiting case. So in the problem without cutouts the complete transverse edges of both the skin and stiffeners were modelled as clamped when using VICONOPT whereas in the problem with cutouts all constraints were removed from the stiffener and from the portions of skin lying within the cutouts.

Figure 3(a) denotes by crosses the nodes at which point supports were present when there were no cutouts. These nodes were equally spaced for all plates of the assembly. It was found that excellent results could be obtained without the expense of constraining all of $\mathrm{u}, \mathrm{v}, \mathrm{w}, \mathrm{dw} / \mathrm{dx}, \mathrm{dv} / \mathrm{dx}$ and $\mathrm{dw} / \mathrm{dy}$ at constrained nodes. Hence Table 2 shows the constraints used, for the cases with and without cutouts, referred to the global axis system shown in Fig. 3(a). The panel was aluminium, subjected to a compressive axial force of $1.16 \mathrm{MN}$, had $l=1.98 \mathrm{~m}$ and had four $\mathrm{T}$ stiffeners spaced as shown on the cross section view of Fig. 3(b).

The panel was first optimised by using VICONOPT to make the local and overall buckling modes for $l=1.8 \mathrm{~m}$ coincident. This optimisation was performed 
without cutouts, using the constraints of Table 2 with approximately $50 \%$ of the nodes shown in Fig. 3(a). The optimised values of the design variables were stiffener height between skin and flange center-lines $=54.20 \mathrm{~mm}$ and thicknesses $=5.06 \mathrm{~mm}$ and 3.10mm for the skin and flanges respectively. The flange breadth $\left(\mathrm{b}_{\mathrm{f}}\right.$, see Fig. 3) was held constant at $30 \mathrm{~mm}$ and the web thickness was constrained to equal half the skin thickness. Offsets ${ }^{2}$ were used to improve the modelling of the structure. The length of this optimised panel was then increased by $10 \%$, i.e. to make $l$ have the correct value of $1.98 \mathrm{~m}$. This procedure ensured that overall buckling governed failure.

\section{Results}

Table 3 shows the decrease in the buckling load factor caused by introducing cutouts. The results shown are for $\xi=1.0$, because $\xi=1.0$ gave lower results than any other $\xi(0 \leq \xi<1.0)$, and are for cutout widths equal to $0 \%, 20 \%, 40 \%$ and $60 \%$ of the stiffener pitch.

The load factors given in Table 3 are converged values obtained by curve extrapolation i.e. best fit quadratic curves, using the points on the plots of load factor against $1 / \mathrm{q}^{2}$ shown in Fig. 4. The results of the quadratic extrapolation are shown on the zero abscissa of Fig. 4. The intercept on the vertical axis is taken as the required load factor because it corresponds to an infinite number of half wavelengths being used. The dashed straight line extrapolations shown in Fig. 4 can be seen to give predictions close to the results of Table 3.

Figure 5 shows $\xi=1$ results for the case with $60 \%$ cutouts and a single additional constraint on each stiffener which prevents $u$ displacement at the 
web/flange junction, and also for the case without cutouts. The best fit quadratic curve extrapolations, shown on the zero abscissa, gave load factors slightly below those of the linear extrapolations shown dashed in Fig. 5, the quadratic extrapolations being 0.838 (i.e. the result of Table 3) for the case without cutouts and 0.809 for the $60 \%$ cutouts case. The introduction of the u constraint causes a large increase in the load factor (i.e. from 0.369 to 0.809 ), so that it becomes close to the result for the case without cutouts (i.e. 0.838).

It can be shown that for the case with no cutouts results for $\xi=0.0$ converge on the clamped result as $\mathrm{q}$ is increased much more quickly than do the $\xi=1$ results. Therefore the converged $\xi=0$ result is shown in brackets in Table 3, from which it can be seen that the $\xi=1$ result of 0.838 extrapolated from Fig. 5 is very accurate, which gives confidence in the other extrapolations of Figs. 4 and 5.

VICONOPT solutions have been validated against finite element based methods for shear loaded panels with simply supported ends ${ }^{5}$. A paper giving solutions for fully clamped ended plates that have been validated against the results of Roark $^{6}$ will shortly be submitted for publication.

\section{Conclusions}

The results clearly show that the presence of a cutout significantly reduces the buckling load of a panel. However the result for the $0 \%$ cutout (i.e. where the stiffener attachment point is still constrained but all its other points are free) also shows a significant decrease. This is due to the transverse line support behaving more like a simple support than a true clamped support. So, irrespective of the size of the 
cutout, leaving the stiffener unconstrained causes the buckling load to drop significantly.

The cutouts results of Fig. 5 clearly show a major increase compared to Fig. 4, with the load factor being quite close to that for the fully clamped case. This has important implications for the fabrication of stiffened panels with transverse supporting structures, namely that it is very beneficial to add to each stiffener web simple extra bracing to prevent displacement in the longitudinal direction at the edge remote from the skin. This gives most of the fabrication advantages of having cutouts while still giving a buckling load close to that of a panel with no cutouts.

The results presented are only for a metal panel with ' $T$ ' stiffeners, but the conclusions are likely to be more generally applicable and can be checked for any given panel by using software such as that used herein.

\section{Acknowledgements}

The first author gratefully acknowledges financial support from the Engineering and Physical Sciences Research Council and British Aerospace.

\section{References}

1)Butler, R. and Williams, F. W., "Optimum Design Using VICONOPT, a Buckling and Strength Constraint Program for Prismatic Assemblies of Anisotropic Plates", Computers and Structures, Vol. 43, No. 4, 1992, pp. 699-708.

2)Wittrick, W. H. and Williams, F. W., "Buckling and Vibration of Anisotropic or Isotropic Plate Assemblies Under Combined Loadings", International Journal of Mechanical Sciences, Vol. 16, No. 4, 1974, pp. 209-239. 
3)Anderson, M. S., Williams, F. W. and Wright, C. J., "Buckling and Vibration of any Prismatic Assembly of Shear and Compression Loaded Anisotropic Plates with an Arbitrary Supporting Structure", International Journal of Mechanical Sciences, Vol. 25, No. 8, 1983, pp. 585-596.

4)Wittrick, W.H. and Williams, F.W., "A General Algorithm for Computing Natural Frequencies of Elastic Structures", Quarterly Journal of Mechanics and Applied Mathematics, Vol. 24, Part 3, 1971, pp. 263-284.

5)Kennedy, D., Williams, F.W. and Anderson, M.S., "Buckling and Vibration Analysis of Laminated Panels Using VICONOPT", Journal of Aerospace Engineering, American Society of Civil Engineers, Vol. 7, No. 3, 1994, pp 245-262.

6)Young, W.C., Roark's Formulas for Stress and Strain, McGraw-Hill, New York, $6^{\text {th }}$ edition, 1989. 
Table 1 Half wavelengths $\lambda$ used in analysis ${ }^{3}$

\begin{tabular}{cc}
\hline \hline$\xi$ & $\lambda$ \\
\hline 0 & $¥, l / 2, l / 4, l / 6, l / 8, \ldots$ \\
0.25 & $4 l,-4 l / 7,4 l / 9,-4 l / 15,4 l / 17, \ldots$ \\
0.5 & $2 l,-2 l / 3,2 l / 5,-2 l / 7,2 l / 9, \ldots$ \\
0.75 & $4 l / 3,-4 l / 5,4 l / 11,-4 l / 13,4 l / 19, \ldots$ \\
1 & $l, l / 3, l / 5, l / 7, l / 9, \ldots$ \\
\hline \hline
\end{tabular}

Table 2 Degrees of freedom constrained at each node for $\xi=1$

\begin{tabular}{ccc}
\hline \hline & With cutouts & Without cutouts \\
\hline flange nodes & - & $\mathrm{u}, \mathrm{dw} / \mathrm{dx}, \mathrm{dv} / \mathrm{dx}$ \\
web only nodes & - & $\mathrm{u}, \mathrm{dw} / \mathrm{dx}$ \\
skin & $\mathrm{u}, \mathrm{w}, \mathrm{dw} / \mathrm{dx}, \mathrm{dv} / \mathrm{dx}$ & $\mathrm{u}, \mathrm{w}, \mathrm{dw} / \mathrm{dx}, \mathrm{dv} / \mathrm{dx}$ \\
& & \\
\hline \hline
\end{tabular}

Table 3 Load factors with and without cutouts

\begin{tabular}{ccccc}
\hline \hline \multirow{2}{*}{$\begin{array}{c}\text { Without } \\
\text { cutouts }\end{array}$} & \multicolumn{4}{c}{ Cutout width as \% of stiffener pitch } \\
\cline { 2 - 5 } & $0 \%$ & $20 \%$ & $40 \%$ & $60 \%$ \\
\hline $0.838(0.841)$ & 0.418 & 0.392 & 0.379 & 0.369 \\
\hline \hline
\end{tabular}




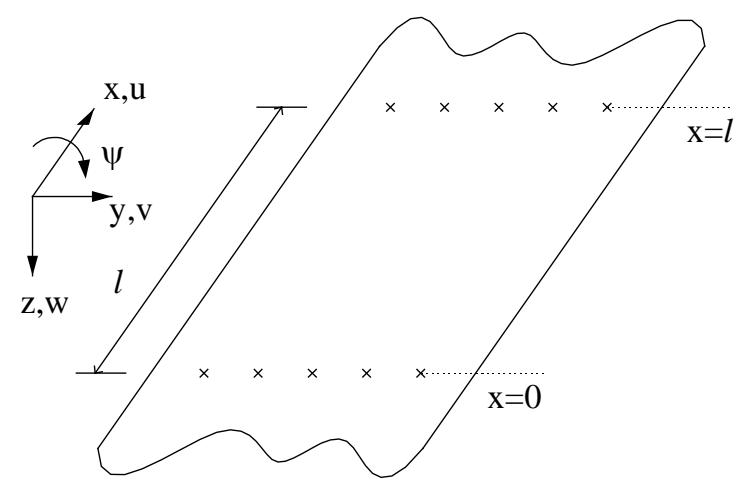

Fig. 1 Plate, showing axis and displacement systems. Crosses denote nodes needed when rigid point supports are used

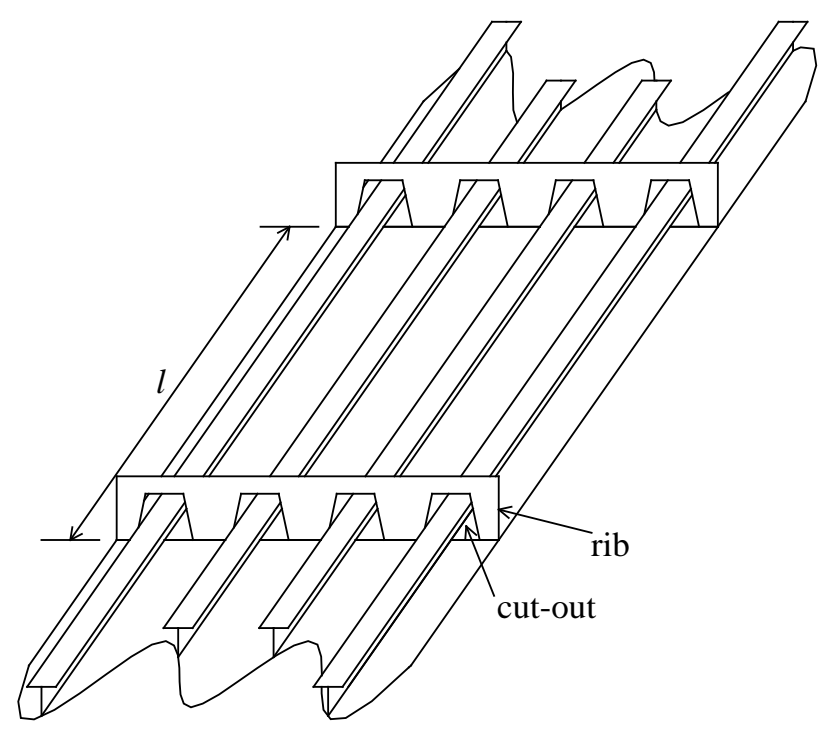

Fig. 2 Panel with ribs and cutouts 
(a)
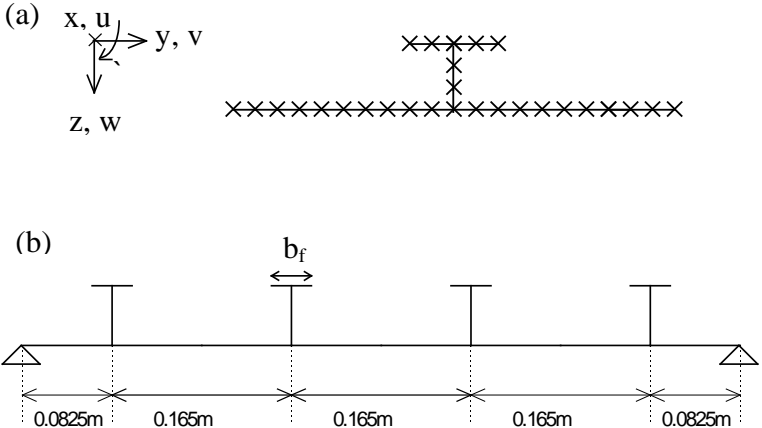

Fig. 3 Cross section of panel studied. (a) Typical $T$ stiffener and associated skin, with the (evenly spaced) crosses denoting nodes at which point supports are present. (b) Complete panel, approximately to scale and with $\Delta$ denoting a continuous line support that enforces $\mathrm{w}=0$

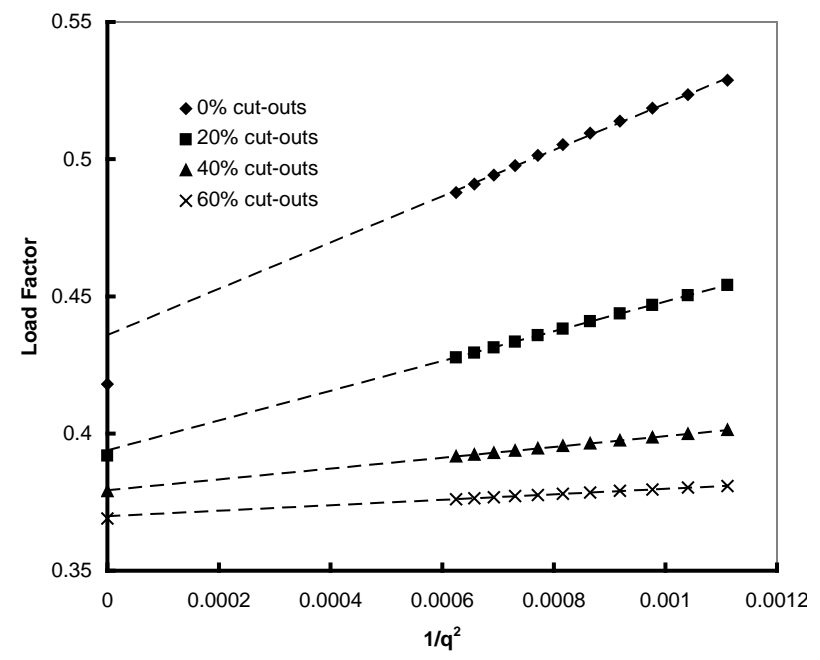

Fig. 4 Graph showing convergence for different sized cutouts, with results of quadratic convergence shown on zero abscissa 


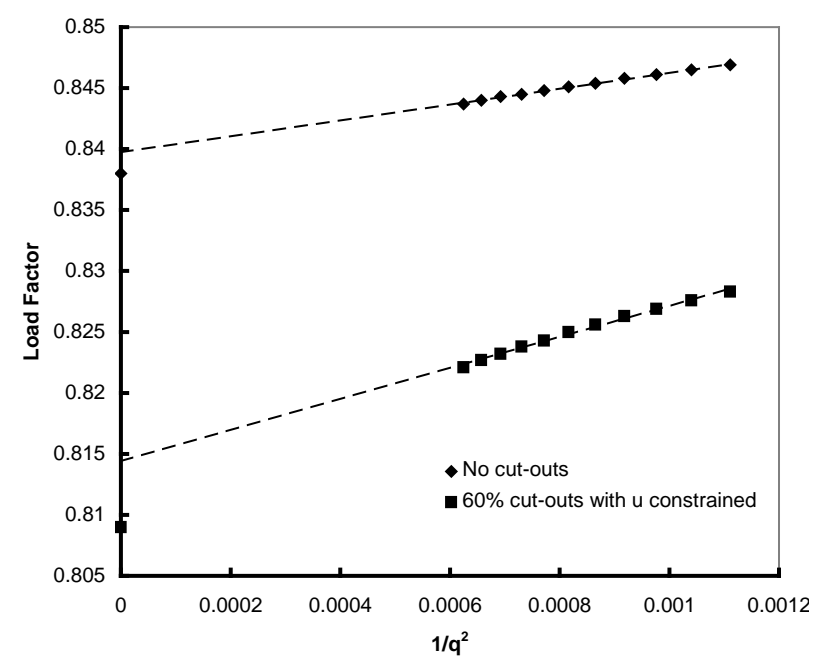

Fig. 5 Effect of constraining $u$ at flange/web junctions compared to case without cutouts, with results of quadratic convergence shown on zero abscissa 


\section{Figure Captions}

Fig. 1 Plate, showing axis and displacement systems. Crosses denote nodes needed when rigid point supports are used

Fig. 2 Panel with ribs and cutouts

Fig. 3 Cross section of panel studied. (a) Typical $\mathrm{T}$ stiffener and associated skin, with the (evenly spaced) crosses denoting nodes at which point supports are present. (b) Complete panel, approximately to scale and with $\Delta$ denoting a continuous line support that enforces $w=0$

Fig. 4 Graph showing convergence for different sized cutouts, with results of quadratic convergence shown on zero abscissa

Fig. 5 Effect of constraining u at flange/web junctions compared to case without cutouts, with results of quadratic convergence shown on zero abscissa 